\title{
B cells: no longer bystanders in liver fibrosis
}

\author{
Rashpal K. Bhogal and Constantin A. Bona
}

Department of Microbiology, Mount Sinai School of Medicine, New York, New York, USA.

\begin{abstract}
Cytokines secreted by cells that mediate the innate and adaptive immune responses play a critical role in regulating the synthesis of ECM components by fibroblasts. Overexpression and deposition of ECM components are dominant features of fibrotic diseases, including hepatic fibrosis. The contribution of $\mathrm{CD}^{+} \mathrm{Th} 2$ cells to hepatic fibrosis has been well described. Now, in this issue of the JCI, Novobrantseva et al. provide data to suggest that hepatic $B$ cells also play a role in liver injury (see the related article beginning on page 3072). In a carbon tetrachloride-induced mouse model of hepatic fibrosis, $T$ cell-deficient mice developed severe liver fibrosis; however, in B cell-deficient animals, hepatic fibrosis was attenuated. This study provides new insight into our understanding of the cells involved in mediating the adaptive immune response that leads to hepatic fibrosis.
\end{abstract}

The ECM is composed of a variety of molecules including the fibrous proteins collagen and fibrillin, adhesion molecules, glycoproteins, proteoglycans, and metalloproteinases, which together form a scaffold on which cells adhere, grow, and differentiate. The most abundant proteins in the ECM belong to the collagen family. In general, ECM molecules are synthesized by fibroblasts; in the liver, they are synthesized by activated hepatic stellate cells (HSCs), portal fibroblasts, and myofibroblasts of bone marrow origin.

Maintenance of the normal structure and function of the ECM involves constant remodeling, which results from regulated, low levels of ECM synthesis and degradation. During wound healing, there is a short-lived shift in the balance of synthesis and degradation, which leads to a transient increase in collagen production and deposition. Chronic collagen production can result in keloid scars.

A permanent imbalance resulting in major alterations in both the quantity and composition of the ECM causes fibrosis. Excessive accumulation of type I collagen is a major pathological feature in diseases such as scleroderma, osteogenesis imperfecta, and scurvy as well as other conditions of the lung, kidney, and liver characterized by tissue fibrosis (1). The main

Nonstandard abbreviations used: $\mathrm{CCl}_{4}$, carbon tetrachloride; CTGF, connective tissue growth factor; HSC, hepatic stellate cell; IH, intrahepatic; TIMP, tissue inhibitor of metalloproteinase; TSK, tight skin.

Conflict of interest: The authors have declared that no conflict of interest exists.

Citation for this article: J. Clin. Invest. 115:2962-2965 (2005). doi:10.1172/JCI26845. causes of liver fibrosis include chronic HCV infection, alcohol abuse, and nonalcoholic steatohepatitis. We note that exposure to carbon tetrachloride $\left(\mathrm{CCl}_{4}\right)$, the most commonly used model of chemically induced hepatic fibrosis in rodents, is not considered to be a main cause of hepatic fibrosis in humans.

The cellular and molecular mechanisms underlying fibrotic disorders are similar regardless of the tissue involved. First, excessive collagen production results from the increased expression of the collagen gene. Second, decreased activity of ECM-removing metalloproteinases, primarily due to the overexpression of tissue inhibitors of metalloproteinases (TIMPs), results in decreased ECM (including collagen) degradation. Third, acquisition of a novel phenotype by fibroblasts is related to abnormal activation of receptors and signaling pathways $(2,3)$, leading to autocrine synthesis of cytokines or growth factors. This finding is supported by the observation that explanted fibroblasts from scleroderma patients retain the new phenotype during multiple serial passages in vitro. Similarly, microarray analyses have revealed high levels of expression of fibrogenic genes, which correlate with prolonged histopathological alteration in a $\mathrm{CCl}_{4}$-induced rat model of liver fibrosis (4).

\section{Fibrosis: an immune-driven process}

Fibroblasts bear receptors that bind various growth factors and cytokines; this triggers specific signaling pathways, leading to activation of transcription factors that bind DNA elements on specific promot- ers and subsequently regulate the expression of genes encoding ECM proteins. The majority of cytokines regulating gene expression in fibroblasts are produced by cells mediating innate or adaptive immunity (Figure 1). Cytokines and growth factors modulating fibroblast function may be grouped into 2 categories: profibrogenic cytokines (e.g., TGF- $\beta 1$, IL-4, IL-13, and IL-6) and growth factors such as PDGF and connective tissue growth factor (CTGF), which are able to increase the synthesis of ECM proteins including collagen. In contrast, proinflammatory cytokines (TNF- $\alpha$, IFN- $\gamma$, and IFN- $\alpha$ ) inhibit collagen gene expression.

TGF- $\beta 1$ is a key mediator of fibrogenesis in various tissues by virtue of its ability to increase the synthesis of ECM proteins and regulate the autocrine synthesis of PDGF and CTGF while it is also able to decrease the synthesis of metalloproteinases by increasing the synthesis of their specific inhibitors (TIMPs) $(3,5,6)$. TGF- $\beta 1$ plays a pivotal role in the evolution of liver fibrosis. Overexpression of the gene encoding TGF- $\beta 1$ in transgenic mice enhances and causes a marked persistence of chemically induced fibrosis (7). Meanwhile, interruption of TGF- $\beta 1$ signaling by delivery of a transgene encoding a dominant-negative, truncated form of TGF- $\beta$ receptor II causes a dramatic reduction of dimethylnitrosamineinduced liver fibrosis in rats (8).

IL- 4 and IL-13 secreted by CD4+ Th2 cells, NKT cells, and mast cells stimulate collagen production subsequent to their binding to corresponding receptors on fibroblasts (Figure 1). The role of $\mathrm{CD} 4^{+} \mathrm{Th} 2$ cell-and STAT6-mediated signaling in the development of fibrosis is well documented in studies performed in animal models, such as in the tight skin (TSK) mouse. TSK mice develop a scleroderma-like syndrome in which cutaneous fibrosis is due to a mutation in the gene encoding fibrillin-1 (9). Targeted mutation of CD4, STAT6, IL-4R $\alpha$, and IL-4 can prevent the occurrence of cutaneous fibrosis (10). Furthermore, an inhibitor of IL-13 has been shown to block the development of hepatic fibrosis (11). Therefore, Th2 cytokine-dominated 


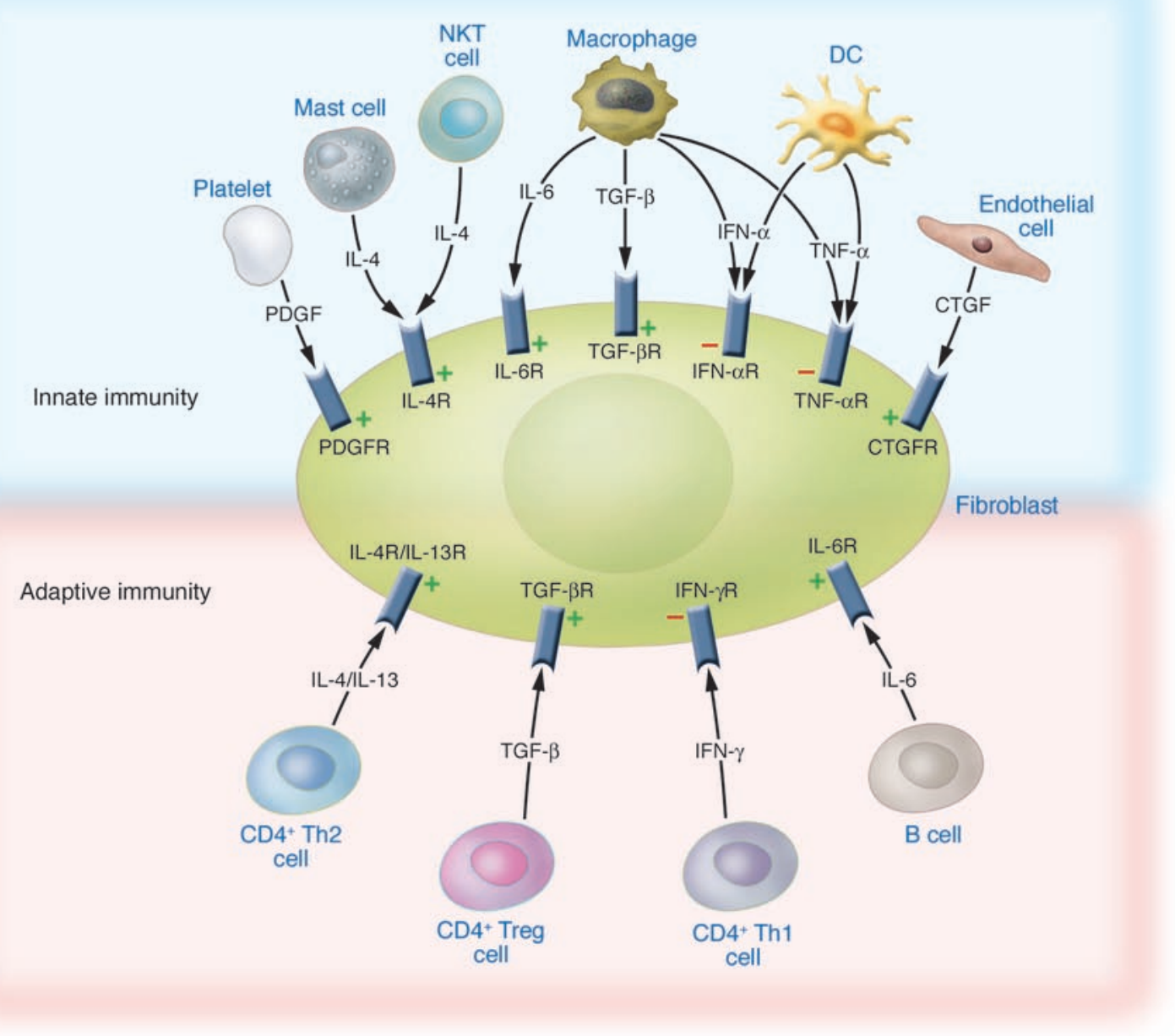

\section{Figure 1}

Effects of cytokines produced by cells mediating innate and adaptive immune responses on the expression of collagen genes in fibroblasts. $\mathrm{R}$, receptor.

responses play a crucial role in the pathogenesis of a variety of fibrotic diseases.

The role of $\mathrm{B}$ cells in the pathogenesis of TSK syndrome has not yet been elucidated. This notion is supported by the following observations: (a) TSK mice unable to produce Igs $\left(\mathrm{TSK}^{+/+} \mathrm{JD}^{-/-}\right.$) do not make autoantibodies but develop cutaneous hyperplasia (12). (b) While the adoptive transfer of TSK $\mathrm{B}$ cells into $\mathrm{MHC}$ matched normal mice induces the synthesis of autoantibodies, transferred TSK B cells do not cause skin sclerosis (13). (c) Moreover, the deletion of CD19, a critical B cell surface-signaling molecule, in TSK mice abolishes the production of autoantibodies, displaying a minimal effect on skin sclerosis (14). Therefore, although B cells may not solely cause cutaneous hyperplasia, they may contribute to its development via interacting with other factors.
Various members of the PDGF family also play a role in wound healing and fibrosis. PDGF-BB is a potent mitogen for fibroblasts, including HSCs, and induces the synthesis of ECM proteins. Furthermore, steatosis and fibrosis in PDGF-C transgenic mice have been shown to be preceded by activation and proliferation of HSCs (15).

\section{B cells and fibrotic diseases}

Autoimmune diseases are classified into systemic and organ-specific diseases. Organ-specific diseases are caused by pathogenic autoantibodies specific for an epitope borne by self antigens expressed by a single cell type. The pathogenic autoantibodies produced by the B2 subset of B cells are defined by several criteria. The most faithful of these criteria is the induction of tissue damage or disease fol- lowing experimental or maternal transfer of these autoantibodies and their isolation from target tissues (16). In systemic autoimmune diseases, autoantibodies are directed against a multitude of self antigens, but only a few fulfill the criteria of pathogenic autoantibodies. For example, anti-RO antibodies cause neonatal systemic lupus erythematosus in humans (16), and passive transfer of serum from $\mathrm{K} / \mathrm{BxN}$ TCR mice to healthy animals induces many of the features of human rheumatoid arthritis (17). The role of $B$ cells in autoimmune disease is not limited to the production of autoantibodies since B cells are also involved in antigen presentation and the production of various cytokines.

In this issue of the JCI, Novobrantseva et al. (18) report that $\mathrm{B}$ cells play a role in $\mathrm{CCl}_{4}$-induced hepatic fibrosis in mice 
in an antibody- and $\mathrm{T}$ cell-independent manner. The authors demonstrate that approximately half of the murine intrahepatic (IH) lymphocyte population comprised functionally operational B cells. Analyses of the VDJ rearrangements of adult murine IH B cells revealed extensive nontemplated (N,P) nucleotide additions in contrast to the few insertions observed in neonatal B cells, strongly suggesting that liver B cells belong to the B2 subset and represent a true IH B cell population. $\mathrm{CCl}_{4}$ stimulates an inflammatory response that includes a TGF- $\beta$-induced increase in type I collagen production $(7,8)$. To examine the role of B cells in liver injury and repair, Novobrantseva et al. studied B cell-deficient, $\mathrm{CCl}_{4}$-treated mice and found that collagen deposition in these mice was decreased in comparison with $\mathrm{CCl}_{4}$-treated WT BALB/c mice. $B$ cell-deficient mice were also able to clear apoptotic cells faster than WT animals. Hence, it was presumed that in the absence of B cells, the ability of macrophages to clear expiring hepatocytes was enhanced. As T cell-deficient mice showed similar levels of collagen deposition when compared with their WT counterparts, the role of $\mathrm{T}$ cells in $\mathrm{CCl}_{4}$ induced liver fibrosis was not considered to be imperative. Finally, the authors examined whether $B$ cell regulation of hepatic fibrosis required Ig production. Mice with normal B cell numbers that lack Ig in their serum or have low Ig levels were found to develop a similar degree of $\mathrm{CCl}_{4}$-induced liver fibrosis when compared with WT mice, indicating that B cells have an impact on the development of liver fibrosis in an antibody-independent manner in this disease model.

\section{The mechanism of B cell involvement in liver fibrosis remains elusive}

There may be several mechanisms by which B cells have an impact on liver fibrosis. First, B cells produce the profibrotic cytokine IL-6, which may contribute to liver fibrosis by its ability to induce the differentiation of HSCs into myofibroblasts, induce fibroblast proliferation, and increase collagen and TIMP synthesis (19). IL-6 levels are increased in human chronic hepatic diseases as well as $\mathrm{CCl}_{4}$-induced hepatic fibrosis (19). As suggested by Novobrantseva et al. (18), chronic administration of $\mathrm{CCl}_{4}$ induces substantial necrosis/apoptosis leading to accumulation of macrophages that remove dying hepatocytes and stimulate fibroblast function. Activated macrophages and fibroblasts could contribute to increased IL- 6 synthesis, thereby increasing collagen synthesis. This is in agreement with the observation that chronic administration of IL- 6 induces hepatic inflammation, increased synthesis of collagen, and fibrosis (20). The fact that $\mathrm{CCl}_{4}$-mediated necrosis and apoptosis in mice is actually reduced in IL-6-deficient mice would support the notion that IL-6 promotes fibrosis (21). This effect may be due to downregulation of MMP2, a metalloproteinase that mediates collagen degradation (22).

Second, alterations in $\mathrm{T}$ cell function in $B$ cell-deficient mice may contribute to the observed impact of B cells on liver fibrosis. Progressive $\mathrm{CCl}_{4}$-induced liver fibrosis in $\mathrm{BALB} / \mathrm{c}$ mice is associated with increased levels of IL-4 and decreased levels of IFN- $\gamma$, synthesized by CD4 $4^{+}$Th 2 and $\mathrm{CD}^{+}$Th 1 cells, respectively (23). As described above, these cytokines have a profound regulatory role in collagen gene expression. Therefore, it is not out of the question that dysfunction of $\mathrm{CD}^{+} \mathrm{T}$ cells in $\mathrm{B}$ cell-deficient mice may contribute to attenuated progression of $\mathrm{CCl}_{4}$-induced fibrosis.

Finally, another plausible explanation for the attenuated fibrosis observed in B cell-deficient mice is that the lack of autoantibody production may affect profibrogenic cytokine activity. It has been demonstrated that anti-fibrillin autoantibodies are present in TSK mice and scleroderma patients (24). Fibrillin-1 is localized in the walls of portal connective tissue, and its expression is increased during fibrosis (25). Therefore, one may consider that anti-fibrillin autoantibodies are present and play a role in liver fibrosis. It was recently shown that anti-fibrillin-1 autoantibodies from scleroderma patients may play a pathogenic role by increasing the transcription and production of ECM components in normal human fibroblasts via the release of sequestered TGF- $\beta 1$ from fibrillin-1 (26).

In conclusion, the study by Novobrantseva et al. (18) offers insight into the mechanisms driving hepatic fibrosis, particularly regarding the contribution of $\mathrm{B}$ cells to liver tissue injury and repair.

Address correspondence to: Constantin A. Bona, Department of Microbiology, Annenberg Building 16-60, Mount Sinai
Medical Center, 1 Gustave L. Levy Place, 1190 Fifth Avenue, New York, New York 10029, USA. Phone: (212) 241-6924; Fax: (212) 423-0711; E-mail: constantin. bona@mssm.edu.

1. Liu, B., and Connolly, M.K. 1998. The pathogenesis of cutaneous fibrosis. Semin. Cutan. Med. Surg. 17:3-11.

2. Strehlow, D., and Korn, J.H. 1998. Biology of the scleroderma fibroblast. Curr. Opin. Rheumatol. 10:572-578.

3. Uitto, J., Bauer, E.A., and Eisen, A.Z. 1979. Scleroderma: increased biosynthesis of triple-helical type I and type III procollagens associated with unaltered expression of collagenase by skin fibroblasts in culture. J. Clin. Invest. 64:921-930.

4. Jiang, Z., You, D.Y., Chen, X.C., and Wu, J. 1992. Monitoring of serum markers for fibrosis during $\mathrm{CCl}_{4}$-induced liver damage. Effects of anti-fibrotic agents. J. Hepatol. 16:282-289.

5. Trojanowska, M., LeRoy, E.C., Eckes, B., and Krieg, T. 1998. Pathogenesis of fibrosis: type 1 collagen and the skin. J. Mol. Med. 76:266-274.

6. Ghosh, A.K. 2002. Factors involved in the regulation of type I collagen gene expression: implication in fibrosis. Exp. Biol. Med. 227:301-314.

7. Shek, F.W., and Benyon, R.C. 2004. How can transforming growth factor beta be targeted usefully to combat liver fibrosis? Eur. J. Gastroenterol. Hepatol. 16:123-126.

8. Sudo, K., Yamada, Y., Moriwaki, H., Saito, K., and Seishima, M. 2005. Lack of tumor necrosis factor receptor type 1 inhibits liver fibrosis induced by carbon tetrachloride in mice. Cytokine. 7:236-244.

9. Siracusa, L.D., et al. 1996. A tandem duplication within the fibrillin 1 gene is associated with the mouse tight skin mutation. Genome Res. 6:300-313.

10. McGaha, T.L., and Bona, C.A. 2002. Role of profibrogenic cytokines secreted by $\mathrm{T}$ cells in fibrotic processes in scleroderma. Autoimmun. Rev. 1:174-181.

11. Chiramonte, M.G., Donaldson, D.D., Cheever, A.W., and Wynn, T.A. 1999. An IL-13 inhibitor blocks the development of hepatic fibrosis during a T-helper type 2-dominated inflammatory response. J. Clin. Invest. 104:777-785.

12. Kasturi, K.N., et al. 1997. B-cell deficiency does not abrogate development of cutaneous hyperplasia in mice inheriting the defective fibrillin-1 gene. J. Autoimmun. 10:505-517.

13. Phelps, R.G., Daian, C., Shinobu, S., Fleischmayer, R., and Bona, C.A. 1993. Induction of skin fibrosis and autoantibodies by infusion of immunocompetent cells from tight-skin mice in $\mathrm{pa} / \mathrm{pa}$ mice. J. Autoimmun. 6:701-718.

14. Sato, S., Fujimoto, M., Hasegawa, M., Takehara, K., and Tedder, T.F. 2004. Altered B lymphocyte function induces systemic autoimmunity in systemic sclerosis. Mol. Immunol. 41:1123-1133.

15. Campbell, J.S., et al. 2005. Platelet-derived growth factor $C$ induces liver fibrosis, steatosis, and hepatocellular carcinoma. Proc. Natl. Acad. Sci. U. S. A. 102:3389-3394.

16. Bona, C.A., and Stevenson, F. 2004. B cells producing pathogenic autoantibodies. In Molecular biology of B cells. F. Alt, T. Honjo, and M. Neuberger, editors. Elsevier. Amsterdam, The Netherlands/Boston, Massachusetts, USA. 381-401.

17. Matsumoto, I., Staub, A., Benoist, C., and Mathis, D. 1999. Arthritis provoked by linked T and B cell recognition of a glycolytic enzyme. Science. 286:1732-1735.

18. Novobrantseva, T.I., et al. 2005. Attenuated liver fibrosis in the absence of B cells. J. Clin. Invest. 115:3072-3082. doi:10.1172/JCI24798.

19. Xue, H., McCauley, R.L., and Zhang, W. 2000. 
Elevated interleukin- 6 expression in keloid fibroblasts. J. Surg. Res. 89:74-77.

20. Choi, I., Kang, H.S., Yang, Y., and Pyun, K.H. 1994. IL-6 induces hepatic inflammation and collagen synthesis in vivo. Clin. Exp. Immunol. 95:530-535.

21. Natsume, M., et al. 1999. Attenuated liver fibrosis and depressed serum albumin levels in carbon tetrachloride-treated IL-6-deficient mice. J. Leukoc. Biol. 66:601-608

22. Bansal, M.B., et al. 2005. Interleukin-6 protects hepatocytes from $\mathrm{CCl}_{4}$-mediated necrosis and apoptosis in mice by reducing MMP-2 expression. J. Hepatol. 42:548-556

23. Bot, A. 1996. Immunoglobulin deficient mice generated by gene targeting as models for studying the immune response. Int. Rev. Immunol. 13:327-340.

24. Bona, C.A. 2000. Anti-fibrillin autoantibodies in scleroderma and mixed connective tissue disease. In Autoantigens and autoantibodies: diagnostic tools and clues to understanding autoimmunity: report on the 5th
Dresden Symposium on Autoantibodies held in Dresden on October 18-21, 2000. K. Conrad, R.-L. Humbel, and M. Meurer, editors. Pabst Science Publishers. Lengerich, Germany. 637-647.

25. Dubuisson, L., et al. 2001. Expression and cellular localization of fibrillin- 1 in normal and pathological human liver. J. Hepatol. 34:514-522.

26. Zhou, X., et al. 2005. Autoantibodies to fibrillin-1 activate normal human fibroblasts in culture through the TGF-b pathway to recapitulate the 'scleroderma phenotype'. J. Immunol. 175:4555-4560.

\title{
CD36 may determine our desire for dietary fats
}

\author{
Nada A. Abumrad \\ Department of Medicine, Division of Nutritional Science, Washington University School of Medicine, St. Louis, Missouri, USA.
}

\begin{abstract}
There is a strong link between high fat intake and obesity. In addition to its high caloric density, dietary fat has a hyperphagic effect, in part as a result of its high palatability. The recent identification by Laugerette et al. of CD36 as a taste receptor for fatty acids provides insight into the molecular basis of our preference for fat (see the related article beginning on page 3177). As we gain more information regarding the function of this receptor, we may be able to devise better strategies to address the addictive potential of dietary fat.
\end{abstract}

The eighteenth-century French philosopher Charles De Montesquieu once commented, "Lunch kills half of Paris, supper the other half." The potential of food consumption to lead to serious health complications is well known and has been extensively studied. The last decade has witnessed impressive progress related to some of the molecular mechanisms underlying the development of nutrition-related pathologies such as obesity, type 2 diabetes, and cardiovascular disease. Information on interorgan cross-talk, on various adipokines and myokines, and on proteins involved in controlling energy intake, storage, or expenditure has greatly enhanced our insight into how the body maintains homeostasis $(1,2)$. Dysfunction within 1 or more homeostatic mechanisms can occur as a result of complex interactions between genetic predisposition and today's affluent lifestyle, often leading to serious health consequences. This has been highlighted by numerous studies of genetically altered animals and by the identification of polymorphisms in humans (3-5).

Nonstandard abbreviations used: FA, fatty acid; SR-BI, scavenger receptor type B, class I.

Conflict of interest: The author has declared that no conflict of interest exists.

Citation for this article: J. Clin. Invest. 115:2965-2967 (2005). doi:10.1172/JCI26955.
A potential new frontier in nutrition research is the examination of how the orosensory experience of food can impact food intake and processing as well as the development of long-term addictive patterns. In addition to food abundance, current enhancements in food palatability through high sugar or fat content further challenge our ability to control food intake and maintain body weight homeostasis. The sensory experience of food can be a primary reinforcer of intake. To what extent food perception is determined by genes versus the environment is a topic that has received limited attention. There is evidence that obesity may be associated with an abnormal brain response to the sensory perception of a meal. This abnormal response may even persist in postobese individuals, creating a high risk of relapse (6). There is little doubt that as individuals, we greatly differ in our ability to experience food at the basic level of taste. According to the NIH, approximately $25 \%$ of Americans are nontasters, $50 \%$ are medium tasters, and $25 \%$ are supertasters. So what are the factors that contribute to determining our food perception, and how are they reflected in what we choose to eat and how much? These questions are important, since our food choices greatly impact body weight outcome in terms of how big and how fast. Impressive progress has been recently accomplished in the identification of taste receptors for various sensations such as sweet, salty, and bitter (7), and this is beginning to contribute insight into the interaction between heredity and the environment in determining food preferences and intake patterns $(8,9)$.

\section{Why do we like fat?}

An exciting new development is the identification of a taste receptor for fat that specifically recognizes fatty acids (FAs), as reported in this issue of the JCI by Laugerette et al. (10). It seems a propos that the report is by a group of French researchers from the University of Bourgogne in Dijon, an area with a rich gastronomic tradition. Dietary fat is particularly addictive, and its excessive intake is strongly linked to obesity. Orosensory perception is thought to play an important role in the spontaneous preference for fat-rich food exhibited by humans and rodents. A hyperphagic effect of a diet with high fat content has been documented and is manifested in increased meal size and decreased intermeal interval (11). Postingestive effects of fat, which include feelings of contentment and satiety and possibly elevation in endogenous opiate levels, also promote long-term preference and positive reinforcement. These effects are not observed with equally palatable, but nondigestible, fat substitutes (12).

Existence of an orosensory receptor for FAs would necessitate a revision of currently held concepts related to food perception. Textbooks still state that taste buds recognize 5 basic sensations; sweet, sour, bitter, salty, and umami (L-amino acid). Evidence presented in several earlier publications strongly suggested that the 